\title{
Prognostic Value of ALDH1, EZH2 and Ki-67 in Astrocytic Gliomas
}

\author{
Shimaa AHMED', Hayam RASHED', Abdelmonem HEGAZY², Abdel Motaleb MOHAMED², \\ Wael ELMESALLAMY ${ }^{4}$
}

Department of ${ }^{1}$ Pathology, ${ }^{2}$ Anatomy and Embryology, ${ }^{3}$ Clinical Oncology and Nuclear Medicine and ${ }^{4}$ Neurosurgery, Zagazig University, Faculty of Medicine, ZAGAZIG, EGYPT

\begin{abstract}
Objective: Tumor stem cells have been found in a variety of neoplasms and stated to have a role in tumor progression. This study aimed to evaluate the prognostic significance of biomarkers which are said to be related to these cells, i.e., EZH2, ALDH1 and Ki-67, and their correlation with each other in astrocytic gliomas.
\end{abstract}

Material and Method: Formalin-fixed, paraffin-embedded tissue specimens of 40 patients with astrocytic glioma who underwent initial surgery during the period from December 2011 to May 2014 at Zagazig University Hospitals were enrolled in the study. Consecutive 4- $\mu \mathrm{m}$ thick sections from formalin-fixed, paraffin-embedded tissue blocks were prepared and stained with hematoxylin and eosin for histopathological evaluation. Immunohistochemical analysis using ALDH1, EZH2 and Ki-67 antibodies were performed to examine the cases.

Results: A total of forty patients; 22 males and 18 females were studied. The lesions were classified as follows: 14 cases of low-grade astrocytoma (WHO grade I or II), 11 cases of anaplastic astrocytoma (WHO grade III), and 15 glioblastomas (WHO grade IV). There was a significant increase in ALDH1 immunoreactivity with increasing the grade of astrocytoma (mean $\pm \mathrm{SD}=0.2 \pm 0.4,0.5 \pm 0.6,1.1 \pm 1.3$ and $2.95 \pm 2.97$ in grade $\mathrm{I}$ to IV astrocytic gliomas, respectively). This expression was significantly correlated with overall survival (OS) and progression-free survival (PFS) $(\mathrm{P}=0.004)$. EZH2 expression was also significantly associated with advanced grades (mean $\pm \mathrm{SD}=1.35 \pm 0.4,3.1 \pm 2.6,7.2 \pm 3.5$ and $9.9 \pm 4.1$, in grade I to IV astrocytic gliomas, respectively). EZH2 and Ki-67 expressions were found to be correlated with OS and PFS (P < 0.001).

Conclusion: Increased expression of ALDH1, EZH2 and KI67 are found to be associated with unfavourable prognosis in patients with astrocytic gliomas and may predict therapeutic modalities.

Key Words: Astrocytoma, Immunohistochemistry, ALDH1, EZH2, Ki-67

\section{INTRODUCTION}

Gliomas represent about $80 \%$ of primary malignant tumors in the central nervous system and rank the first among brain tumors (1). CNS neoplasms comprise about $3 \%$ of primary malignant tumors in Egypt. They are the most common solid tumor in children (2). Astrocytic gliomas represent a heterogeneous group of malignancies which are classified into low grade astrocytomas (grade I-II), anaplastic astrocytomas (grade III) and glioblastoma (GB) (grade IV) (3). GB is the most common and aggressive primary brain tumor in humans. $52 \%$ of primary brain malignancies and $20 \%$ of all primary intracranial tumors in the United States are GBs (4). Patients with astrocytic gliomas have poor survival despite the recent advances in management. There have been only a minimal advance in prognosis especially in GB with a very short median survival (5).

(Turk Patoloji Derg 2016, 32:70-81)

Received : 07.08.2015 Accepted : 02.10.2015
Brain tumors are thought to originate from a small population of undifferentiated cells having characteristics of normal neural stem cells. These cells have been named after brain tumor stem cells, responsible for tumor initiation and therapy resistance in primary brain tumors (6).

Aldehyde dehydrogenase 1 (ALDH1) is a detoxifying enzyme that catalyzes oxidation of intracellular aldehydes, leading to production of retinoic acid from retinol, which has a major role in cell proliferation and differentiation and maintenance of stem cell phenotype. ALDH1 is needed for resistance to alkylating agent through its detoxifying effect $(7,8)$. This biomarker has been detected in stem cells of both normal and neoplastic tissues (9).

Polycomb group (PcG) proteins have a major role in regulating several physiological and pathological activities, such as oncogenesis (10). PcG protein complexes are

Correspondence: Abdelmonem HEGAZY

Department of Anatomy and Embryology, Zagazig University,

Faculty of Medicine, ZAGAZIG, EGYPT

E-mail: dr.abdelmonemhegazy@yahoo.com Phone: +00 20552289888 
grouped into complex 1 (PRC1) and complex 2 (PRC2) (11). Enhancer of zeste homolog 2 (EZH2) is the core member of PRC2 (12). EZH2 acts through activating Ras and the nuclear factor (NF)- $\kappa B$ pathway to control cell oncogenesis through regulation of transforming growth factor $-\beta 1$ expression and functions (13). Also, EZH2 interposes histone methylation and recruits DNA methyl transferase in the silencing of many genes, related to cell cycle control and survival $(14,15)$. Also, it is considered as a recent biomarker of unfavorable prognosis in cases with ependymoma (16).

Ki-67 protein is expressed in all active phases of the cell cycle. It represents an excellent biomarker applicated in many neurosurgery centers to predict aggressiveness of gliomas and patients' outcomes $(17,18)$.

The current study aimed to evaluate the prognostic role and predictive value of ALDH1, EZH2 and Ki-67 in astrocytic gliomas.

\section{MATERIAL and METHODS}

Patients and Tissue Specimens: Formalin-fixed, paraffinembedded (FFPE) tissue specimens of 40 patients with astrocytomas who underwent initial surgery during the period from December 2011 to May 2014 at Zagazig University Hospitals were enrolled in our study and were obtained from the archives of the Pathology Department, Zagazig University. Clinical information and detailed follow-up data for all patients were taken from medical files. All examined tumors were located in the cerebral hemispheres; recurrent tumors were excluded. None of the patients had received chemotherapy or radiotherapy prior to surgery.

Follow-up information obtained from the clinical records ranged from 6 to 36 months. Preoperative Karnofsky performance status (KPS) score was obtained from the neurological examination data. The study complied with the guidelines of Zagazig University ethics committee. MRI repeated every 3-4 months was used as standard practice to detect patients' response. A complete response (CR) was defined as the complete absence of enhancing or nonenhancing tumor; partial response (PR) was a shrinkage of $>50 \%$ of the tumor area; stable disease (SD) was defined as a decrease of tumor area from 0 to $50 \%$ with stable or reduced steroid dosage; and minor response (MR) as a reduction of $>25 \%$ but $<50 \%$ of the tumor area. An increase of $>25 \%$ of the tumor area was designated as progressive disease (PD).

Histopathologic Examination: Ten tissue specimens were obtained by stereotactic brain biopsies. Subtotal resection was done in 11 cases. In the remaining 19 cases, gross total resection was performed. All tissue samples were fixed with neutral $4 \%$ formaldehyde solution. Consecutive $4-\mu \mathrm{m}$ thick sections from formalin-fixed, paraffin-embedded tissue blocks were prepared and stained with hematoxylin and eosin (H\&E) for histopathological classification (3).

Immunohistochemical Staining: FFPE tissues were cut into $4-\mu \mathrm{m}$ thick sections and transferred to positively charged slides. Then, sections were subjected to dewaxing, rehydration, blocking with hydrogen peroxide, and antigen retrieval (Dako target retrieval solution, citrate buffer $\mathrm{pH}$ 6.0) with microwave. The slides were then incubated overnight at $2-8{ }^{\circ} \mathrm{C}$ with primary antibodies: anti-ALDH1 rabbit monoclonal antibody (Abcam, Cambridge, UK; dilution 1:100); EZH2 (BD Biosciences, CA, 11/EZH2; dilution 1:100); and Ki-67 antibody (Dako, Glostrup, Denmark, clone MIB-1; dilution 1:50). Incubation with secondary antibody and product visualization (Dako) was performed with diaminobenzidine substrate as the chromogen. The slides were finally counterstained with Mayer's hematoxylin and washed once each with distilled water and PBS.

Specimens of infiltrating duct carcinoma of the breast, liver and tonsillar tissue were used as positive controls for EZH2, ALDH1 and Ki-67 antibodies, respectively. Negative controls, obtained by replacement of primary antibodies by PBS, were included within each slide batch.

\section{Assessment of Immunohistochemical Staining Results}

Evaluation of ALDH1 Immunostaining: The degree of reactivity was carried out by evaluation of the percentage of cells stained per section. Sections were graded as ALDH1negative (no staining), $<2 \%$ ALDH1-positive and $\geq 2 \%$ ALDH1-positive (14).

Evaluation of EZH2 Immunostaining: Positive-nuclear staining cells (nuclear staining) for EZH2 in ten highpower microscopic fields were counted and the percentage of positive cells was calculated. Scoring was as follows: 0 (0\%), 1 (1-10\%), $2(11-50 \%)$ and $3(>50 \%)$. Also, the staining intensity was scored as: 0 (negative), 1 (weak), 2 (moderate) and 3 (strong). A final score was obtained for each case by multiplying the two scores. A final score of $\geq 4.5$ was considered to be high (19).

Evaluation of Ki-67 Immunostaining: Ki-67 index was calculated as the percentage of immunoreactive nuclei per 200 cells in five high power fields. Staining of $0-10 \%$ and $\geq 10 \%$ cell nuclei was considered to be low and high indices, respectively (20). 
Statistical Analysis: Continuous variables were expressed as the mean $\pm \mathrm{SD}$ and median (range), and the categorical variables were expressed as numbers (percentages). Continuous variables were checked for normality by using Shapiro-Wilk test. Mann Whitney U test was used to compare between two groups of non-normally distributed variables while the Kruskal-Wallis $\mathrm{H}$ test was used for more than two groups. Percentages of categorical variables were compared using the Pearson's Chi-square $\left(\chi^{2}\right)$ test. Spearman's rank correlation coefficient was calculated to assess correlations between EZH2, ALDH1 and Ki-67; (+) sign was indicator for direct relationship and (-) sign was indicator for inverse relationship. Values near 1 were indicators for strong relationship and values near 0 were indicators for weak relationship. Overall Survival (OS) was calculated as the time from surgery to death or the most recent follow-up contact (censored). Recurrence free survival (RFS) was calculated as the time from complete response to recurrence or date of last known recurrent free status(censored). Stratification of OS and PFS was done according to all clinicopathological parameters. These time-to-death and time-to-recurrence distributions were estimated using the method of Kaplan-Meier plot, and compared using two-sided exact log-rank test. All tests were two sided. A $P$-value $<0.05$ was considered significant. All statistics were performed using SPSS 22.0 for Windows (SPSS Inc., Chicago, IL, USA) \& MedCalc Windows (MedCalc Software bvba 13, Ostend, Belgium).

\section{RESULTS}

Patients and Their Clinicopathological Parameters: Our study included forty patients; 22 males and 18 females, with an age range from 7 to 72 years (mean: $40.2 \pm 20.2$ ). The lesions were classified as low-grade $[5$ pilocytic astrocytomas (WHO I) and 9 diffuse astrocytomas (WHO II); and 26 were classified as high-grade gliomas [11 anaplastic astrocytomas (WHO III)] and 15 glioblastomas (WHO IV)]. The clinicopathological data and the treatment regimen of the patients are shown in Table I.

Table I: Clinicopathological features of 40 patients with astrocytic glioma

\begin{tabular}{|c|c|c|c|c|c|}
\hline Features & WHO I & WHO II & WHO III & WHO IV & P-value \\
\hline No. of patients & 5 & 9 & 11 & 15 & \\
\hline $\begin{array}{l}\text { Age (years): } \\
\text { Mean } \\
\text { Median } \\
\text { Range }\end{array}$ & $\begin{array}{c}13 \pm 3.8 \\
14 \\
7-17\end{array}$ & $\begin{array}{c}30.4 \pm 15.3 \\
32 \\
9-50\end{array}$ & $\begin{array}{c}45.1 \pm 17.1 \\
52 \\
12-63\end{array}$ & $\begin{array}{c}51.3 \pm 17.7 \\
53 \\
11-72\end{array}$ & $0.001^{\star \star}$ \\
\hline $\begin{array}{l}\text { Gender: } \\
\text { Male (22) } \\
\text { Female (18) }\end{array}$ & $\begin{array}{c}2(9.1 \%) \\
3(16.7 \%)\end{array}$ & $\begin{array}{l}5(22.7 \%) \\
4(22.2 \%)\end{array}$ & $\begin{array}{l}6(27.3 \%) \\
5(27.8 \%)\end{array}$ & $\begin{array}{l}9(40.9 \%) \\
6(33.3 \%)\end{array}$ & 0.895 \\
\hline $\begin{array}{l}\text { KPS } \\
>80 \\
<80 \\
\end{array}$ & $\begin{array}{c}4(22.2 \%) \\
1(4.5 \%) \\
\end{array}$ & $\begin{array}{c}7(38.9 \%) \\
2(9.1 \%) \\
\end{array}$ & $\begin{array}{l}3(16.7 \%) \\
8(36.4 \%)\end{array}$ & $\begin{array}{c}4(22.2 \%) \\
11(50.0 \%)\end{array}$ & $0.02^{*}$ \\
\hline $\begin{array}{l}\text { Size } \\
\quad \leq 35 \mathrm{~mm} \\
>35 \mathrm{~mm}\end{array}$ & $\begin{array}{c}5(22.2 \%) \\
0(0.0 \%)\end{array}$ & $\begin{array}{c}7(38.9 \%) \\
2(9.1 \%)\end{array}$ & $\begin{array}{l}5(27.8 \%) \\
6(27.3 \%)\end{array}$ & $\begin{array}{c}1(5.6 \%) \\
14(63.6 \%)\end{array}$ & $0.0002^{\star \star}$ \\
\hline $\begin{array}{l}\text { Surgery } \\
\text { Gross total resection } \\
\text { Subtotal resection } \\
\text { Biopsy } \\
\end{array}$ & $\begin{array}{c}5(26.3) \\
0(0.0) \\
0(0.0) \\
\end{array}$ & $\begin{array}{l}9(47.4) \\
0(0.0) \\
0(0.0) \\
\end{array}$ & $\begin{array}{l}5(26.3) \\
5(45.5) \\
1(10.0) \\
\end{array}$ & $\begin{array}{l}0(0.0) \\
6(54.5) \\
9(90.0) \\
\end{array}$ & $0.001^{\star *}$ \\
\hline $\begin{array}{l}\text { Adjuvant treatment } \\
\text { No } \\
\text { Radiotherapy } \\
\text { Radiotherapy and chemotherapy } \\
\text { Combination }\end{array}$ & $\begin{array}{c}5(100.0) \\
0(0.0) \\
0(0.0)\end{array}$ & $\begin{array}{c}8(88.9) \\
1(11.1) \\
0(0.0)\end{array}$ & $\begin{array}{c}3(27.3) \\
8(72.7) \\
0(0.0)\end{array}$ & $\begin{array}{c}0(0.0) \\
4(26.7) \\
11(73.3)\end{array}$ & $<0.001^{\star *}$ \\
\hline Median overall survival (months) & $34(33-36)$ & $32(28-36)$ & $21(14-23)$ & $10(4-11)$ & $0.001^{\star \star}$ \\
\hline Median progression free survival (months) & $34(33-36)$ & $32(20-36)$ & $18(12-23)$ & $7(1-10)$ & $0.001^{* *}$ \\
\hline
\end{tabular}

Continuous variables were expressed as the mean \pm SD \& median (range); categorical variables were expressed as a number (percentage).; Qualitative data are presented as number (\%); $\ddagger$ Kruskal Wallis $\mathrm{H}$ test $₫$ Chi-square test; $P<0.05$ is significant. 
Association Between Astrocytic Glioma Grade and Clinicopathological Parameters: WHO grade of astrocytic gliomas was correlated to age, size, extent of tumor resection, type of adjuvant treatment, PFS, KPS and OS $(P$ $<0.05)$, but insignificantly associated with patient $\operatorname{sex}(P=0$. 895) (Table I).

\section{Association Between ALDH1 Expression and} Clinicopathological Parameters: During follow-up period, 26 of the 40 patients had recurrent glioma and 20 patients had died. ALDH1-positivity $>2 \%$ was associated with short PFS and OS significantly $(P=0.001)$ (Figures $1 \mathrm{~A}-\mathrm{D}, 2 \mathrm{~A}-$ D). ALDH1 was cytoplasmic in distribution (Figure 3AC). This expression was significantly higher in astrocytic glioma (mean: $1.5 \pm 2.2$ ) than non-neoplastic brain tissue (mean: 0$)(P=0.003)$. There was significant difference between ALDH1 immunoreactivity and different grades of astrocytic glioma ( $P=0.014$ ) (Table II). ALDH1 expression was significantly associated with KPS $(P<0.001)$, however it was not associated with age, sex or tumor size and type of tumor resection (Table III).

Association Between EZH2 Expression and Clinicopathological Parameters: EZH2 were expressed in the nuclei (Figure 4A-D). This expression was significantly higher in astrocytic glioma (mean: $6.6 \pm 4.7)$ than nonneoplastic brain tissue (mean: $0.8 \pm 0.2)(P<0.0001)$. There was significant difference between EZH2 immunoreactivity among different grades of astrocytic glioma $(P<0.001)$ (Table II). EZH2 expression was significantly associated with age, size, KPS and type of resection $(P=0.05)$. However, no significant association between EZH2 expression and sex was found ( $P=0.94$ ) (Table III). EZH2 overexpression was also significantly associated with poor OS and PFS $(P$ $<0.0001$ ) (Figures 1A-D, 2A-D).
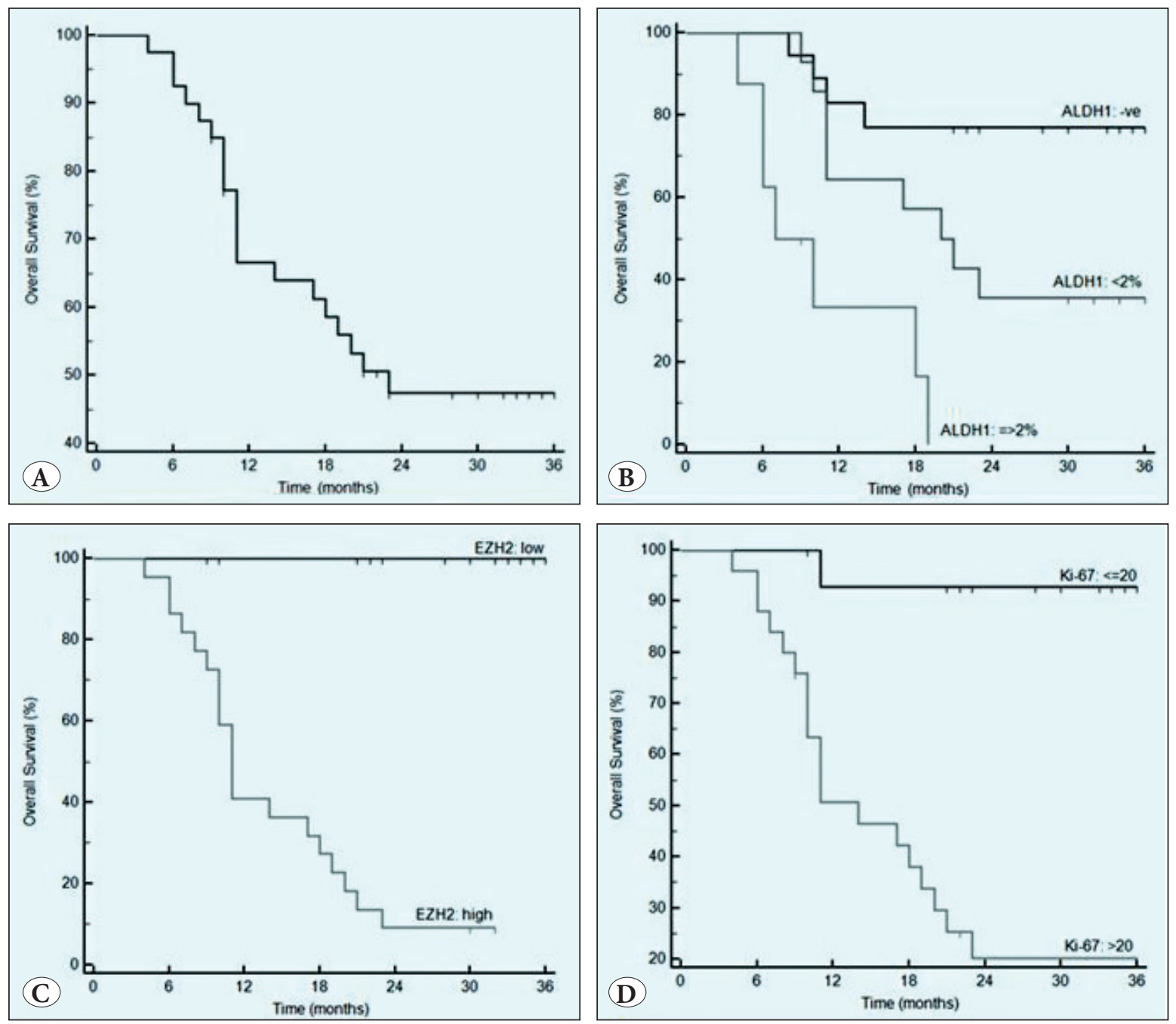

Figure 1: Kaplan-Meier plot of survival: A) In all studied astrocytic glioma patients (N=40), B) Stratified according to ALDH1 expression, C) Stratified according to EZH2 expression, D) Stratified according to Ki-67 expression. 
Table II: Relation between different grades of astrocytic glioma\&clinicopathological parameters in patients $(\mathrm{n}=40)$

\begin{tabular}{|c|c|c|c|c|c|}
\hline \multirow{2}{*}{ Characteristics } & Grade I & Grade II & Grade III & Grade IV & \multirow{2}{*}{$\mathbf{P}$} \\
\hline & $(n=5)$ & $(n=9)$ & $(n=11)$ & $(n=15)$ & \\
\hline \multicolumn{6}{|l|}{ ALDH1 } \\
\hline Mean \pm SD & $0.2 \pm 0.4$ & $0.5 \pm 0.6$ & $1.1 \pm 1.3$ & $2.95 \pm 2.97$ & \multirow{2}{*}{0.014} \\
\hline Median (Range) & $0(0-1)$ & $0(0-1.2)$ & $1(0-4)$ & $1.6(0-9)$ & \\
\hline \multicolumn{6}{|l|}{\begin{tabular}{|l|} 
EZH2 \\
\end{tabular}} \\
\hline Mean \pm SD & $1.5 \pm 0.4$ & $3.1 \pm 2.6$ & $7.2 \pm 3.5$ & $9.9 \pm 4.1$ & \multirow{2}{*}{$<0.001$} \\
\hline Median (Range) & $1.7(1-2)$ & $2.2(1-9)$ & $8(3-12)$ & $10(3-17)$ & \\
\hline
\end{tabular}

Qualitative data are presented as number $(\%)$; Kruskal Wallis $\mathrm{H}$ test $₫$ Chi-square test; $P<0.05$ is significant.
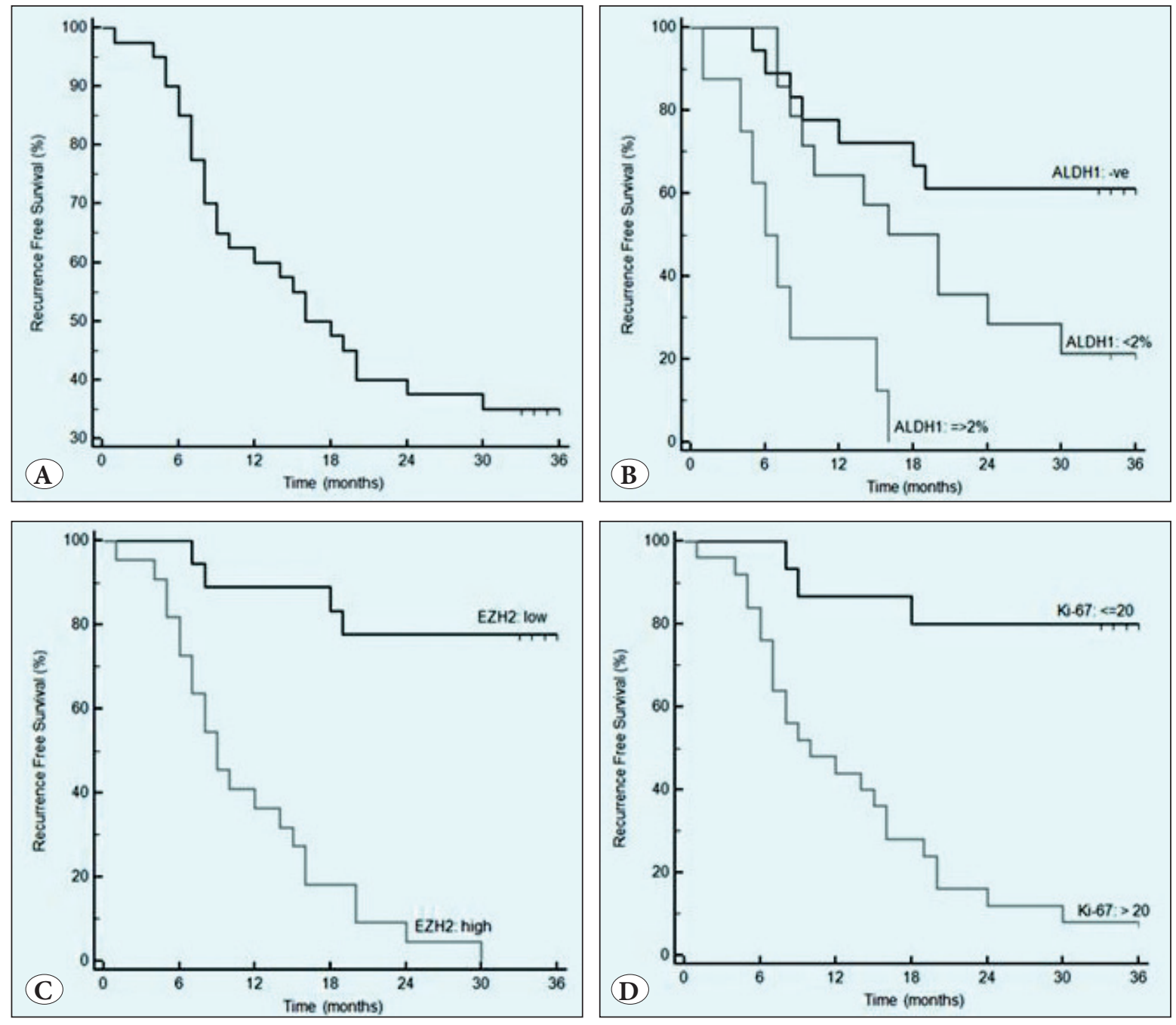

Figure 2: Kaplan-Meier plot of recurrence: A) In all studied astrocytic glioma patients (N=40), B) Stratified according to ALDH1 expression, C) Stratified according to EZH2 expression, D) Stratified according to Ki-67 expression. 
Table III: Relation between ALDH1, EZH2 and KI67 with the clinicopathological parameters in patients $(\mathrm{n}=40)$

\begin{tabular}{|c|c|c|c|c|c|c|c|c|c|c|c|}
\hline \multirow[b]{2}{*}{ Characteristics } & \multirow[b]{2}{*}{ n (\%) } & \multicolumn{3}{|c|}{ ALDH1 } & \multirow[b]{2}{*}{$\mathbf{P S}$} & \multicolumn{2}{|c|}{ EZH2 } & \multirow[b]{2}{*}{$\mathbf{P S}$} & \multicolumn{2}{|c|}{ Ki-67 } & \multirow[b]{2}{*}{$\mathbf{P S}$} \\
\hline & & $\begin{array}{c}(-) \\
(n=18)\end{array}$ & $\begin{array}{c}<2 \% \\
(n=14)\end{array}$ & $\begin{array}{l}\geq 2 \% \\
(n=8)\end{array}$ & & $\begin{array}{c}\text { Low } \\
(n=18)\end{array}$ & $\begin{array}{c}\text { High } \\
(n=22)\end{array}$ & & $\begin{array}{c}\leq 10 \\
(n=15)\end{array}$ & $\begin{array}{c}>10 \\
(n=25)\end{array}$ & \\
\hline \multicolumn{12}{|l|}{ Age (years) } \\
\hline$\leq 45$ years & $20(50 \%)$ & $\begin{array}{c}10 \\
(55.6 \%)\end{array}$ & $\begin{array}{c}8 \\
(57.1 \%)\end{array}$ & $2(25 \%)$ & \multirow{2}{*}{0.285} & $\begin{array}{c}13 \\
(72.2 \%)\end{array}$ & $\begin{array}{c}7 \\
(31.8 \%)\end{array}$ & \multirow{2}{*}{0.011} & $\begin{array}{c}11 \\
(73.3 \%)\end{array}$ & $9(36 \%)$ & \multirow{2}{*}{0.022} \\
\hline$>45$ years & $20(50 \%)$ & $\begin{array}{c}8 \\
(44.4 \%)\end{array}$ & $\begin{array}{c}6 \\
(42.9 \%)\end{array}$ & $6(75 \%)$ & & $\begin{array}{c}5 \\
(27.8 \%)\end{array}$ & $\begin{array}{c}15 \\
(68.2 \%)\end{array}$ & & $\begin{array}{c}4 \\
(26.4 \%)\end{array}$ & $\begin{array}{c}16 \\
(64 \%)\end{array}$ & \\
\hline \multicolumn{12}{|l|}{ Sex } \\
\hline Male & $22(55 \%)$ & $\begin{array}{c}10 \\
(55.6 \%) \\
\end{array}$ & $\begin{array}{c}8 \\
(57.1 \%) \\
\end{array}$ & $4(50 \%)$ & \multirow{2}{*}{0.947} & $\begin{array}{c}10 \\
(55.6 \%) \\
\end{array}$ & $\begin{array}{c}12 \\
(54.5 \%) \\
\end{array}$ & \multirow{2}{*}{0.949} & $\begin{array}{c}10 \\
(66.7 \%) \\
\end{array}$ & $\begin{array}{c}12 \\
(48 \%) \\
\end{array}$ & \multirow{2}{*}{0.251} \\
\hline Female & $18(45 \%)$ & $\begin{array}{c}8 \\
(44.4 \%)\end{array}$ & $\begin{array}{c}6 \\
(42.9 \%)\end{array}$ & $4(50 \%)$ & & $\begin{array}{c}8 \\
(44.4 \%)\end{array}$ & $\begin{array}{c}10 \\
(45.5 \%)\end{array}$ & & $\begin{array}{c}5 \\
(33.3 \%)\end{array}$ & $\begin{array}{c}13 \\
(52 \%) \\
\end{array}$ & \\
\hline \multicolumn{12}{|l|}{ Tumor size } \\
\hline$\leq 3.5 \mathrm{~cm}$ & $18(45 \%)$ & $9(50 \%)$ & $\begin{array}{c}6 \\
(42.9 \%) \\
\end{array}$ & $\begin{array}{c}3 \\
(37.5 \%) \\
\end{array}$ & \multirow{2}{*}{0.823} & $\begin{array}{c}12 \\
(66.7 \%) \\
\end{array}$ & $\begin{array}{c}6 \\
(27.3 \%) \\
\end{array}$ & \multirow{2}{*}{0.013} & $\begin{array}{c}10 \\
(66.7 \%) \\
\end{array}$ & $8(32 \%)$ & \multirow{2}{*}{0.033} \\
\hline$>3.5 \mathrm{~cm}$ & $22(55 \%)$ & $9(50 \%)$ & $\begin{array}{c}8 \\
(57.1 \%)\end{array}$ & $\begin{array}{c}5 \\
(62.5 \%)\end{array}$ & & $\begin{array}{c}6 \\
(33.3 \%)\end{array}$ & $\begin{array}{c}16 \\
(72.7 \%)\end{array}$ & & $\begin{array}{c}5 \\
(33.3 \%)\end{array}$ & $\begin{array}{c}17 \\
(68 \%) \\
\end{array}$ & \\
\hline \multicolumn{12}{|c|}{ Performance status } \\
\hline$<80$ & $22(55 \%)$ & $\begin{array}{c}4 \\
(22.2 \%) \\
\end{array}$ & $\begin{array}{c}11 \\
(78.6 \%) \\
\end{array}$ & $\begin{array}{c}7 \\
(87.5 \%) \\
\end{array}$ & \multirow{2}{*}{0.001} & $\begin{array}{c}3 \\
(16.7 \%) \\
\end{array}$ & $\begin{array}{c}19 \\
(86.4 \%)\end{array}$ & \multirow{2}{*}{$<0.001$} & $0(0 \%)$ & $\begin{array}{c}22 \\
(88 \%) \\
\end{array}$ & \multirow{2}{*}{$<0.001$} \\
\hline$\geq 80$ & $18(45 \%)$ & $\begin{array}{c}14 \\
(77.8 \%) \\
\end{array}$ & $\begin{array}{c}3 \\
(21.4 \%) \\
\end{array}$ & $\begin{array}{c}1 \\
(12.5 \%) \\
\end{array}$ & & $\begin{array}{c}15 \\
(83.3 \%) \\
\end{array}$ & $\begin{array}{c}3 \\
(13.6 \%) \\
\end{array}$ & & $\begin{array}{c}15 \\
(100 \%) \\
\end{array}$ & $3(12 \%)$ & \\
\hline \multicolumn{12}{|l|}{ Resection } \\
\hline $\begin{array}{l}\text { Gross total } \\
\text { resection }\end{array}$ & $\begin{array}{c}19 \\
(47.5 \%) \\
\end{array}$ & $\begin{array}{c}10 \\
(55.6 \%) \\
\end{array}$ & $7(50 \%)$ & $2(25 \%)$ & \multirow{3}{*}{0.101} & $\begin{array}{c}13 \\
(72.2 \%) \\
\end{array}$ & $\begin{array}{c}6 \\
(27.3 \%) \\
\end{array}$ & \multirow{3}{*}{0.017} & $\begin{array}{c}11 \\
(73.3 \%) \\
\end{array}$ & $8(32 \%)$ & \multirow{3}{*}{0.009} \\
\hline $\begin{array}{l}\text { Subtotal } \\
\text { resection }\end{array}$ & $\begin{array}{c}11 \\
(27.5 \%) \\
\end{array}$ & $\begin{array}{c}5 \\
(27.8 \%) \\
\end{array}$ & $\begin{array}{c}5 \\
(35.7 \%) \\
\end{array}$ & $\begin{array}{c}1 \\
(12.5 \%)\end{array}$ & & $\begin{array}{c}3 \\
(16.7 \%) \\
\end{array}$ & $\begin{array}{c}8 \\
(36.4 \%) \\
\end{array}$ & & $\begin{array}{c}4 \\
(26.7 \%) \\
\end{array}$ & $7(28 \%)$ & \\
\hline Biopsy & $10(25 \%)$ & $\begin{array}{c}3 \\
(16.7 \%)\end{array}$ & $\begin{array}{c}2 \\
(14.3 \%)\end{array}$ & $\begin{array}{c}5 \\
(62.5 \%)\end{array}$ & & $\begin{array}{c}2 \\
(11.1 \%)\end{array}$ & $\begin{array}{c}8 \\
(36.4 \%)\end{array}$ & & $0(0 \%)$ & $\begin{array}{c}10 \\
(40 \%) \\
\end{array}$ & \\
\hline \multicolumn{12}{|c|}{ Adjuvant treatment } \\
\hline No & $16(40 \%)$ & $\begin{array}{c}10 \\
(55.6 \%) \\
\end{array}$ & $\begin{array}{c}4 \\
(28.6 \%) \\
\end{array}$ & $2(25 \%)$ & \multirow{3}{*}{0.067} & $\begin{array}{c}13 \\
(72.2 \%)\end{array}$ & $\begin{array}{c}3 \\
(13.6 \%) \\
\end{array}$ & \multirow{3}{*}{$<0.001$} & $\begin{array}{c}11 \\
(73.3 \%) \\
\end{array}$ & $5(20 \%)$ & \\
\hline Radiotherapy & $\begin{array}{c}13 \\
(32.5 \%) \\
\end{array}$ & $\begin{array}{c}6 \\
(33.3 \%) \\
\end{array}$ & $\begin{array}{c}6 \\
(42.9 \%) \\
\end{array}$ & $\begin{array}{c}1 \\
(12.5 \%) \\
\end{array}$ & & $\begin{array}{c}3 \\
(16.7 \%) \\
\end{array}$ & $\begin{array}{c}9 \\
(40.9 \%) \\
\end{array}$ & & $\begin{array}{c}4 \\
(26.7 \%) \\
\end{array}$ & $9(36 \%)$ & 0.001 \\
\hline $\begin{array}{l}\text { Chemoradiot- } \\
\text { herapy }\end{array}$ & $\begin{array}{c}11 \\
(27.5 \%)\end{array}$ & $\begin{array}{c}2 \\
(11.1 \%) \\
\end{array}$ & $\begin{array}{c}4 \\
(28.6 \%) \\
\end{array}$ & $\begin{array}{c}5 \\
(62.5 \%) \\
\end{array}$ & & $\begin{array}{c}2 \\
(11.1 \%) \\
\end{array}$ & $\begin{array}{c}10 \\
(45.5 \%) \\
\end{array}$ & & $0(0 \%)$ & $\begin{array}{c}11 \\
(44 \%) \\
\end{array}$ & \\
\hline Survival & & & & & & & & & & & \\
\hline Died & $20(50 \%)$ & $\begin{array}{c}4 \\
(22.2 \%) \\
\end{array}$ & $\begin{array}{c}9 \\
(64.3 \%) \\
\end{array}$ & $\begin{array}{c}7 \\
(87.5 \%) \\
\end{array}$ & 008 & $0(0 \%)$ & $\begin{array}{c}20 \\
(90.9 \%) \\
\end{array}$ & $<0001$ & $1(6.7 \%)$ & $\begin{array}{c}19 \\
(76 \% \\
\end{array}$ & 0 \\
\hline Alive & $20(50 \%)$ & $\begin{array}{c}14 \\
(77.8 \%)\end{array}$ & $\begin{array}{c}5 \\
(35.7 \%) \\
\end{array}$ & $\begin{array}{c}1 \\
(12.5 \%)\end{array}$ & 0.004 & $\begin{array}{c}18 \\
(100 \%)\end{array}$ & $2(9.1 \%)$ & $<0.001$ & $\begin{array}{c}14 \\
(93.3 \%)\end{array}$ & $6(24 \%)$ & $<0.001$ \\
\hline
\end{tabular}

Qualitative data are presented as number (\%); $₫$ Chi-square test; $\mathrm{P}<0.05$ is significant. Qualitative data are presented as number (\%): NR denote not reached yet.; ${ }^{\star}$ Chi-square test.; $\$$ Log rank test; CI: Confidence interval, HR: hazard ratio; $\mathrm{P}<0.05$ is significant. 

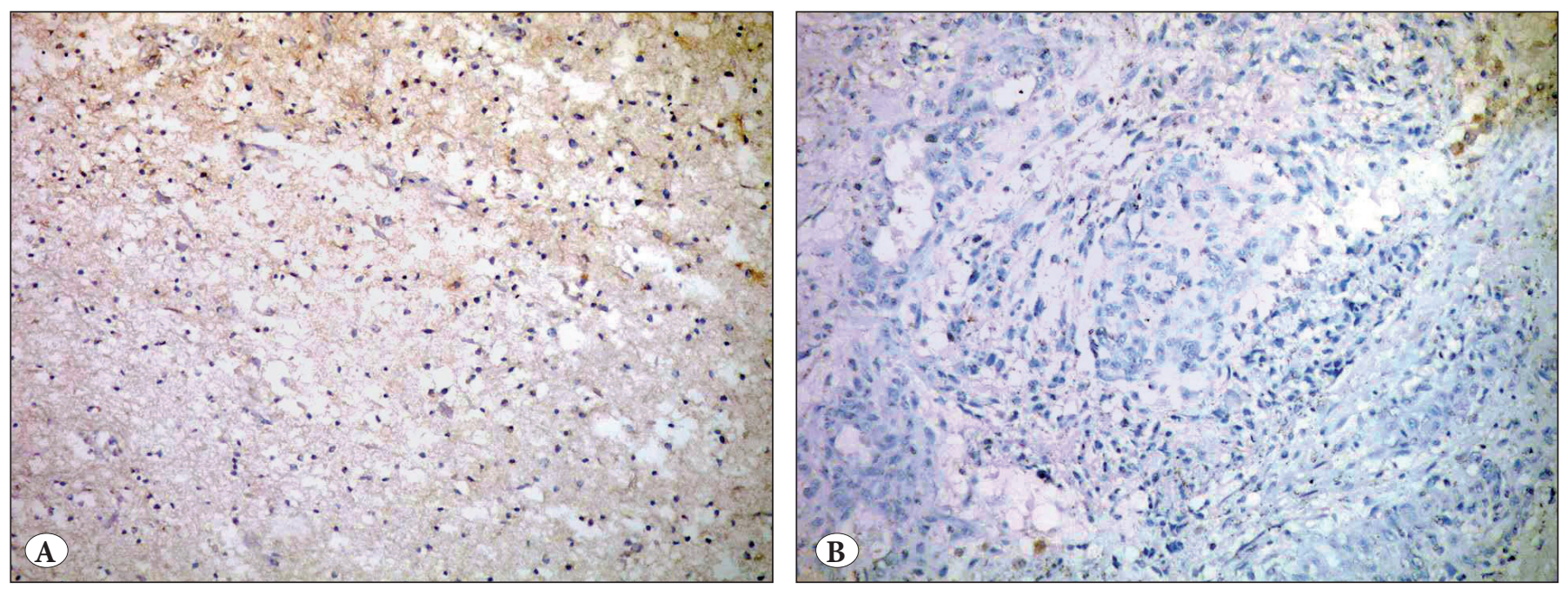

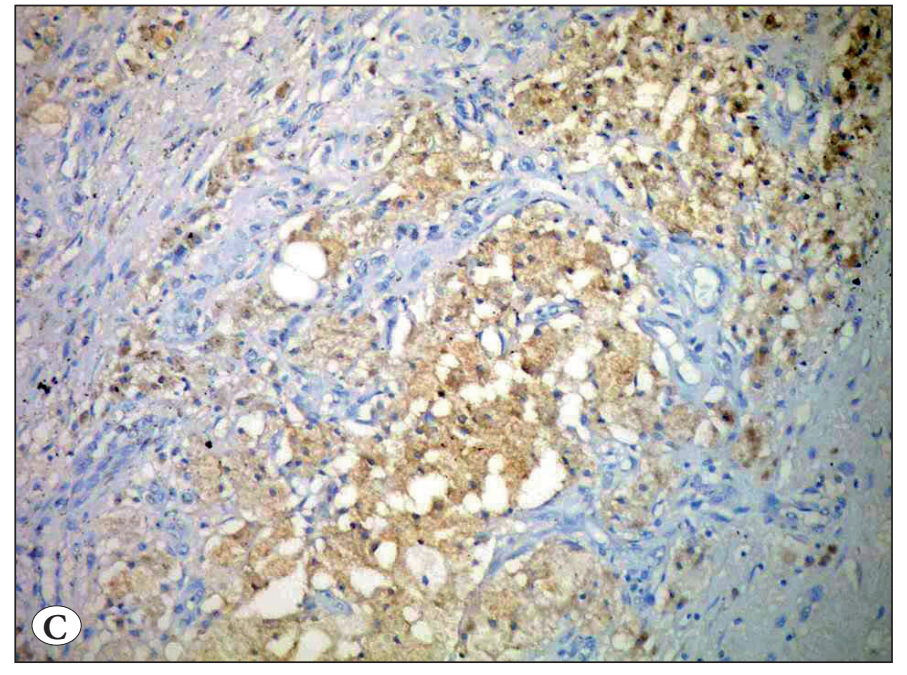

Association Between Ki-67 Expression and Clinicopathological Parameters : Ki-67 was expressed in the nuclei (Figure 5A-D). There was significant difference between Ki-67 index among different grades of astrocytic glioma. High Ki-67 index was significantly associated with poor OS and PFS $(P<0.0001)$ (Figures $1 \mathrm{~A}-\mathrm{D}, 2 \mathrm{~A}-\mathrm{D})$.

Correlation Between ALDH1, EZH2 and Ki-67 in All Studied Astrocytic Glioma Patients: Significant positive correlation was found between ALDH1 and EZH2 ( $\mathrm{r}=$ $+0.598, P<0.0001)$, as well as between ALDH1, EZH2 \& Ki-67 $(P<0.0001)$ (Tables IV,V).

Relation Between ALDH1, EZH2 and Ki-67 and Response to Therapy in All Studied Astrocytic Glioma Patients: There was significant association between ALDH1, EZH2 and Ki-67 and response to therapy. Four patients had no ALDH1 immunoreactivity, low EZH2 and low Ki-67 index. However, high expression of ALDH1, EZH2 and Ki-67 were associated with progressive disease in 22 cases. Partial
Figure 3: A) Low-grade astrocytoma showing low levels of ALDH1 cytoplasmic expression (ALDH1; x200). B) High grade astrocytoma showing low levels of ALDH1 cytoplasmic expression (ALDH1; x200). C) High grade astrocytoma showing high levels of ALDH1 cytoplasmic expression (ALDH1; x200).

response was achieved in 7 patients, while 7 patients had stable disease (Table VI).

\section{DISCUSSION}

Astrocytic gliomas are the most common central nervous system neoplasms. They are heterogeneous tumors at molecular and phenotypical levels (21). Despite the great advances in therapeutic modalities, survival of patients with astrocytic gliomas remains poor, especially with GB, which is a highly aggressive tumor resisting treatment (22). Therefore, better understanding of tumor biology, molecular basis of tumor invasion and spread and resistance to conventional therapies may aid to improve patients' outcome (23).

Tumor stem cells (TSCs) comprise a subpopulation of tumor cells with continuous self-renewal capacity and the ability to initiate tumor growth. TSCs are also responsible for tumor recurrence and resistance to recent therapeutic 
Table IV: Correlation between ALDH1 \& EZH2 in patients $(n=40)$

\begin{tabular}{|l|c|c|c|c|}
\hline \multirow{2}{*}{} & $\mathbf{2}$ & \multicolumn{2}{c|}{ EZH2 } \\
\cline { 2 - 5 } & $\mathbf{r}$ & $\mathbf{p}$ & $\mathbf{r}$ & $\mathbf{p}$ \\
\hline ALDH1 & --- & & +0.598 & $<0.001$ \\
\hline EZH2 & +0.598 & $<0.001$ & --- & \\
\hline
\end{tabular}

C: Spearman's rank correlation coefficient; $P<0.05$ is significant.

Table V: Association between ALDH1, EZH2 \& Ki-67 in patients $(\mathrm{n}=40)$

\begin{tabular}{|l|c|c|c|c|c|c|}
\hline & \multicolumn{2}{|c|}{ ALDH1 } & \multicolumn{2}{c|}{ EZH2 } & \multicolumn{2}{c|}{ Ki-67 } \\
\cline { 2 - 7 } & $\mathbf{C}$ & $\mathbf{p}$ & $\mathbf{C}$ & $\mathbf{p}$ & $\mathbf{C}$ & +0.602 \\
\hline ALDH1 & --- & & +0.514 & 0.001 & +0.001 \\
\hline EZH2 & +0.514 & 0.001 & --- & & +0.601 & $<0.001$ \\
\hline Ki-67 & +0.602 & $<0.001$ & +0.601 & $<0.001$ & $-\cdots$ & \\
\hline
\end{tabular}

C: Contingency coefficient; $P<0.05$ is significant.
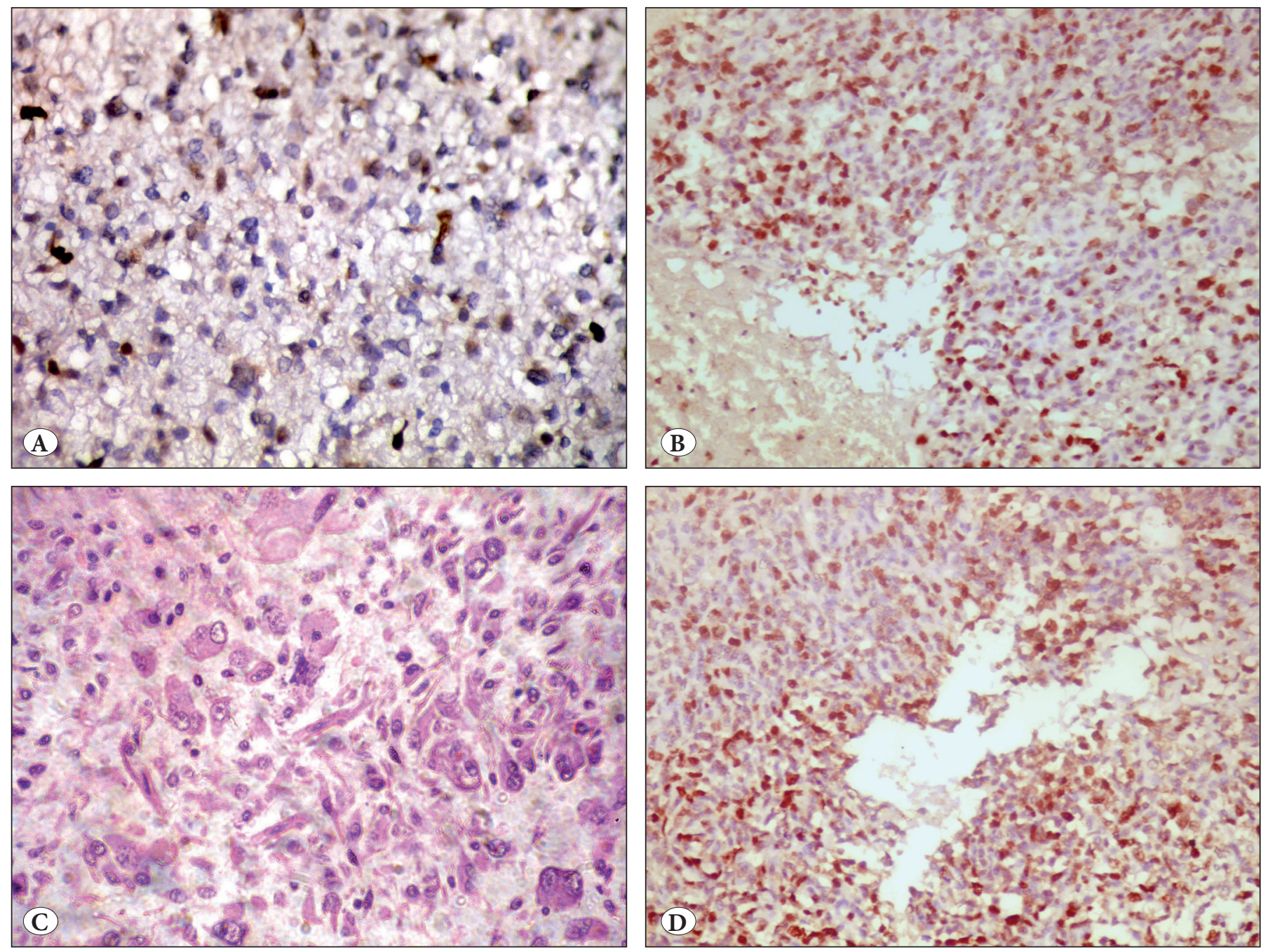

Figure 4: A) Low-grade astrocytoma showing low levels of EZH2 nuclear expression (EZH2; x400). B) High grade astrocytoma showing high levels of EZH2 nuclear expression (EZH2; x100). C) Giant cell glioblastoma showing the presence of giant cells (H\&E; x400). D) Giant cell glioblastoma showing high levels of EZH2 nuclear expression (EZH2; x400). 
Table VI: Relation between ALDH1,EZH2,Ki-67 and type of response in patients $(n=40)$

\begin{tabular}{|c|c|c|c|c|c|c|c|c|c|c|c|}
\hline \multirow[b]{2}{*}{ Response } & \multirow[b]{2}{*}{ No (\%) } & \multicolumn{3}{|c|}{ ALDH1 } & \multirow[b]{2}{*}{$\mathrm{p} \S$} & \multicolumn{2}{|c|}{ EZH2 } & \multirow[b]{2}{*}{$\mathbf{p} \S$} & \multicolumn{2}{|c|}{ Ki-67 } & \multirow[b]{2}{*}{$\mathrm{p} \S$} \\
\hline & & $\begin{array}{c}(-) \\
(n=18)\end{array}$ & $\begin{array}{c}<2 \% \\
(n=14)\end{array}$ & $\begin{array}{l}\geq 2 \% \\
(\mathrm{n}=8)\end{array}$ & & $\begin{array}{c}\text { Low } \\
(n=18)\end{array}$ & $\begin{array}{c}\text { High } \\
(\mathrm{n}=22)\end{array}$ & & $\begin{array}{c}\leq 20 \\
(n=15)\end{array}$ & $\begin{array}{c}>20 \\
(\mathrm{n}=25)\end{array}$ & \\
\hline $\begin{array}{l}\text { Compelet } \\
\text { response }\end{array}$ & $4(10 \%)$ & $\begin{array}{c}4 \\
(22.2 \%)\end{array}$ & $0(0 \%)$ & $0(0 \%)$ & \multirow{4}{*}{0.022} & $4(22.2 \%)$ & $0(0 \%)$ & \multirow{4}{*}{$<0.001$} & $\begin{array}{c}4 \\
(26.7 \%)\end{array}$ & $0(0 \%)$ & \multirow{4}{*}{0.003} \\
\hline $\begin{array}{l}\text { Partial } \\
\text { response }\end{array}$ & $\begin{array}{c}7 \\
(17.5 \%)\end{array}$ & $\begin{array}{c}4 \\
(22.2 \%)\end{array}$ & $\begin{array}{c}3 \\
(21.4 \%)\end{array}$ & $0(0 \%)$ & & $6(33.3 \%)$ & $1(4.5 \%)$ & & $\begin{array}{c}4 \\
(26.7 \%)\end{array}$ & $\begin{array}{c}3 \\
(12 \%)\end{array}$ & \\
\hline Stable disease & $\begin{array}{c}7 \\
(17.5 \%)\end{array}$ & $\begin{array}{c}5 \\
(27.8 \%) \\
\end{array}$ & $\begin{array}{c}2 \\
(14.3 \%) \\
\end{array}$ & $0(0 \%)$ & & $5(27.8 \%)$ & $2(9.1 \%)$ & & $\begin{array}{c}4 \\
(26.7 \%) \\
\end{array}$ & $\begin{array}{c}3 \\
(12 \%)\end{array}$ & \\
\hline $\begin{array}{l}\text { Progressive } \\
\text { disease }\end{array}$ & $22(55 \%)$ & $\begin{array}{c}5 \\
(27.8 \%) \\
\end{array}$ & $\begin{array}{c}9 \\
(64.3 \%) \\
\end{array}$ & $\begin{array}{c}8 \\
(100 \%) \\
\end{array}$ & & $3(16.7 \%)$ & \begin{tabular}{|c|}
19 \\
$(86.4 \%)$ \\
\end{tabular} & & $3(20 \%)$ & $\begin{array}{c}19 \\
(76 \%) \\
\end{array}$ & \\
\hline
\end{tabular}

Qualitative data are presented as number (\%); $₫$ Chi-square test; $P<0.05$ is significant.
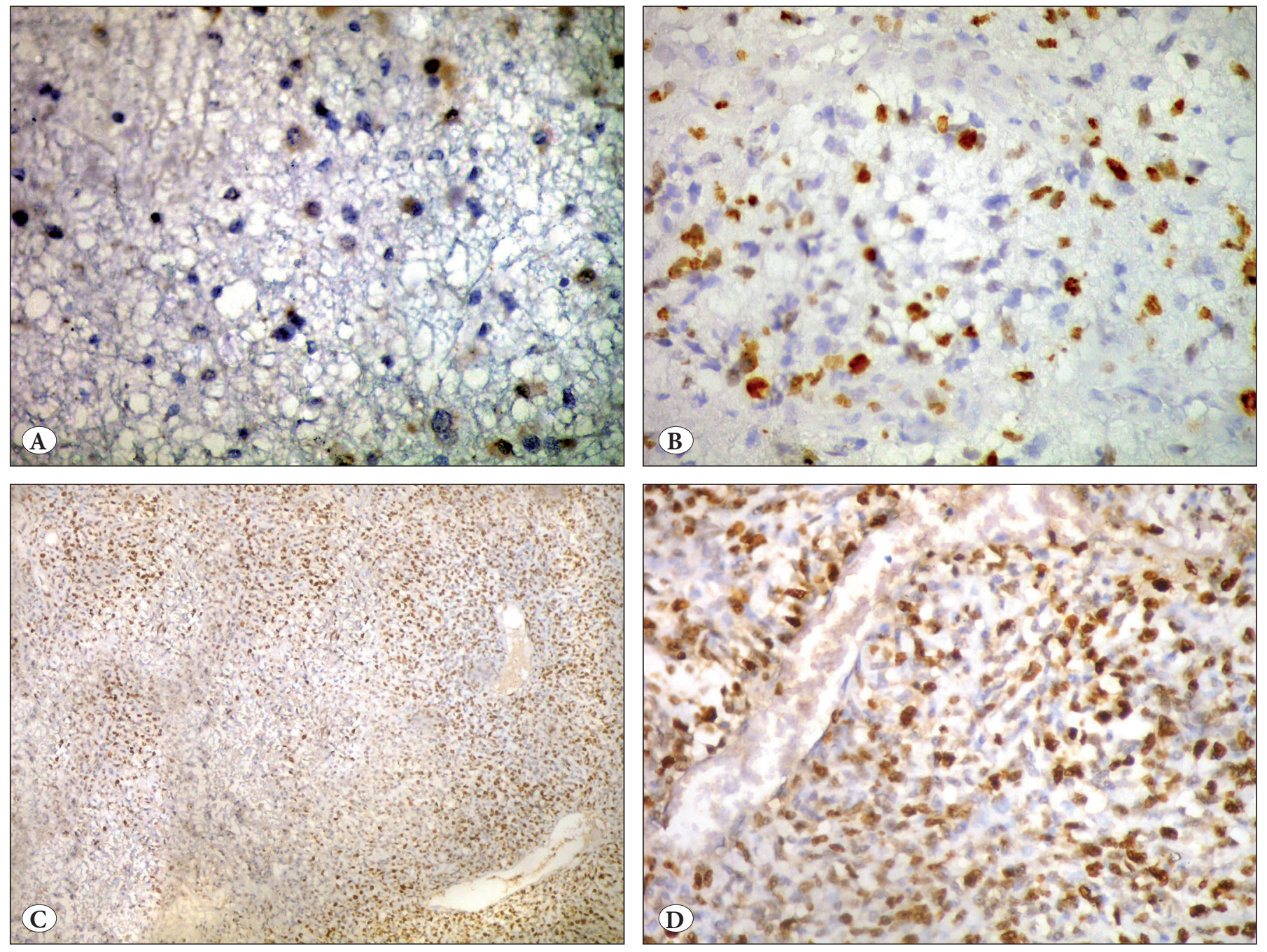

Figure 5: A) Low grade astrocytoma showing low levels of Ki-67 nuclear expression (Ki-67; x400). B) Low grade astrocytoma showing high levels of Ki-67 nuclear expression (Ki-67; x400). C) High grade astrocytoma showing high levels of Ki-67 nuclear expression (Ki-67; x100). D) High grade astrocytoma showing high levels of Ki-67 nuclear expression (Ki-67; x400). 
modalities (24-26). The identification of these cells seems to be crucial for understanding the process of carcinogenesis, and for the development of targeted therapies (27).

ALDH1 is a specific stem cell marker in normal brain tissue and it is suggested to be a novel stem cell marker in GB. High level of ALDH1 in GB cells may be responsible for the presence of undifferentiated stem cells (27). In the current study, ALDH1 expression was found to be increased significantly from grade I to grade II astrocytoma, to anaplastic astrocytoma and glioblastoma (mean $\pm \mathrm{SD}$ : $0.2 \pm 0.4,0.5 \pm 0.6,1.1 \pm 1.3$ and $2.95 \pm 2.97$, respectively). ALDH1 was not detected in low-grade astrocytomas or it was expressed in $<2 \%$ of cells. For the high-grade astrocytic gliomas, $18.2 \%$ of grade III and $40 \%$ of grade IV tumors had $\geq 2 \%$ of ALDH1-positive cells. This leads to the conclusion that the level of ALDH1 is strongly correlated with astrocytic glioma grade and necessarily with the patients' prognosis. Our results go with those of Liu et al. (14), who found that the percentage of ALDH1-positive cells increased with tumor grade and correlated with unfavorable clinical course in patients with astrocytic glioma. All these results stated that TSCs have a major role in the carcinogenesis of glioma. Rasper et al. (27) stated that high level of ALDH1 was detected in $83.3 \%$ of GBs. Moreover, they studied ALDH1 expression in GB cell lines and cell culture and searched for the neurosphere formation, finding that high ALDH1 protein levels improve neurosphere formation. However, there was deficiency in neurosphere formation after ALDH1 inhibition and lack of ALDH1 leads to incomplete maturation of TSCs and improper neurosphere formation (27). ALDH1 is responsible for transformation of retinol to retinoic acid which leads to up-regulation apoptosis and differentiation genes in the GB cell line (28).

Several studies have displayed that the expression of TSCs biomarkers is positively correlated to tumor grade and inversely associated with OS and PFS in glioma cases (29). In the current study, patients with ALDH1-positivity of $\geq 2 \%$ was significantly linked to short PFS and OS as compared to those with positivity of $<2 \%(P=0.003)$. This is in agreement with the results of Liu et al. (14), who found that over-expression of ALDH1 was significantly correlated with short PFS and OS $(P<0.001)$.

EZH2 methylate STAT3, leading to increased STAT3 activity by increasing tyrosine phosphorylation of STAT3, so they promote carcinogenesis of glioblastoma stem-like cells (30). Transcriptional regulation of c-myc by EZH2 involved in glioblastoma cancer stem cell maintenance and carcinogenesis (31).
From the therapeutic point of view, EZH 2-mediated signal loop, EZH2-CDK4/6-pRb-E2F1, which is involved in carcinogenesis of glioma, could be inhibited by the candidate miRNA. MiR-138 is the miRNA that is diminished in GB samples and potent to suppress EZH2 expression through inhibition of EZH2-CDK4/6-pRb-E2F1 signal loop (32).

In the present study, increased expression of EZH2 protein was significantly associated with advanced grades. None of the low-grade astrocytic glioma cases exhibited high EZH2 indices. These results are in accord with those of $\mathrm{Wu}$ et al. (19), who found that the up-regulations of EZH2 proteins was significantly associated with increased grades $(P<$ $0.001)$.

Zhang et al. (33), performed gene expression profiling of glioma and found that the level of EZH2 mRNA increased expressively in high grade gliomas compared to lower grades. Therefore, EZH2 may have a great role in glioma development. Also, overexpression of EZH2 was investigated in metastatic tumors (13). Tumor cell proliferation and progression, and poor outcome in prostate, endometrial, breast cancer and melanoma were found to be related with EZH2 over-expression $(34,35)$.

In our study, a significant association was present between EZH2 over-expression and poor OS and PFS $(P<0.001)$. Patient survival declines significantly in those with high EZH2 expression ( 9 vs. 11 months for both PFS and OS). These results go with that of $\mathrm{Wu}$ et al. (19) who demonstrated that the high level of EZH2 was greatly linked to decreased survival $(P=0.006)$. Also, Zhang et al. (33) stated that GB cases expressing higher levels of EZH2 were linked to shorter survival relative to those with lower levels.

The current study revealed a significant difference between Ki-67 indices among different grades of astrocytic glioma. These results are in a general agreement with previous results declaring that Ki-67 index was found to correlate with tumor grade and clinical behavior $(36,37)$. But, a study performed by Chaloob et al. (38) did not support such significant correlation. The authors attributed this situation to environmental, racial and geographical differences, in addition to the differences in sample size and antibodies used for the detection of Ki-67 antigen in the different studies.

Due to the heterogeneity of astrocytic gliomas, it is likely that only combinations of markers will able to give sufficient sensitivity and specificity to be useful for clinical purposes. In this paper, such a combination with EZH2, 
ALDH1 and Ki-67 in astrocytic gliomas was studied and an expressive association was observed among them. Therefore, over-expression of EZH2 and ALDH1 was significantly correlated with increased cell proliferation as assessed by high Ki-67 index. This suggests that tumors expressing EZH2 and ALDH1 will have unfavourable prognosis, increased recurrence rate, poor response to therapy and poor survival.

In conclusion, expression of ALDH1, EZH2 and Ki-67 was found to be increase significantly with astrocytic glioma progression and increased expression levels were associated with short patient survival. Our data suggested that ALDH1, EZH2 and Ki-67 can be considered as potential biomarkers to detect astrocytic glioma patients with unfavorable outcome, can be targeted for future therapy and may aid to stratify the patients into different risk groups.

\section{REFERENCES}

1. Taylor LP. Diagnosis, treatment, and prognosis of glioma: Five new things. Neurology. 2010;75:S28-32.

2. Zalata K, El-Tantawy D, Abdel-Aziz A, Ibraheim A, Halaka A, Gawish H. Safwat M, Mansour N, Mansour M, Shebl A. Frequency of central nervous system tumors in delta region, Egypt. Indian J Pathol Microbiol. 2011;54:299-306.

3. Louis DN, Ohgaki H,Wiestler OD, Cavenee WK, Burger PC, Jouvet A, Scheithauer BW, Kleihues P. The 2007 WHO classification of tumours of the central nervous system. Acta Neuropathol. 2007;114:97-109.

4. Pointer KB, Clark PA, Zorniak M, Alrfaei BM, Kuo JS. Glioblastoma cancer stem cells. Biomarker and therapeutic advances. Neurochem Int. 2014;71:1-7.

5. Zhang $\mathrm{X}$, Yang $\mathrm{H}$, Gong B, Jiang C, Yang L. Combined gene expression and protein interaction analysis of dynamic modularity in glioma prognosis. J Neurooncol. 2012;107:281-8.

6. Singh SK, Hawkins C, Clarke ID, Squire JA, Bayani J, Hide T, Henkelman RM, Cusimano MD, Dirks PB. Identification of human brain tumour initiating cells. Nature. 2004;432:396-401.

7. Jones RJ, Barber JP, Vala MS, Collector MI, Kaufmann SH, Ludeman SM, Colvin OM, Hilton J. Assessment of aldehyde dehydrogenase in viable cells. Blood. 1995;85:2742-6.

8. Marcato P, Dean CA, Giacomantonio CA, Lee PW. Aldehyde dehydrogenase: Its role as a cancer stem cell marker comes down to the specific isoform. Cell Cycle. 2011;10:1378-84.

9. Choi SA, Lee JY, Phi JH, Wang KC, Park CK, Park SH, Kim SK. Identification of brain tumour initiating cells using the stem cell marker aldehyde dehydrogenase. Eur J Cancer. 2014;50:137-49.

10. Qi L, Cao JL, Hu Y, Yang JG, Ji Y, Huang J, Zhang Y, Sun DG, $\mathrm{Xia}$ HF, Ma X. The dynamics of polycomb group proteins in early embryonic nervous system inmouse and human. Int J Dev Neurosci. 2013;31:487-95.

11. Lanzuolo C, Orlando V. Memories from the polycomb group proteins. Annu Rev Genet. 2012:46:561-89.
12. Ohm J, McGarvey K, Yu X, Cheng L, Schuebel K, Cope L, Mohammad HP, Chen W, Daniel VC, Yu W, Berman DM, Jenuwein T, Pruitt K, Sharkis SJ, Watkins DN, Herman JG, Baylin SB. A stem cell-like chromatin pattern may predispose tumor suppressor genes to DNA hypermethylation and heritable silencing. Nat Genet. 2007;39:237-42.

13. Min J, Zaslavsky A, Fedele G, McLaughlin SK, Reczek EE, De Raedt T, Guney I, Strochlic DE, Macconaill LE, Beroukhim R, Bronson RT,Ryeom S, Hahn WC, Loda M, Cichowski K. An oncogene-tumor suppressor cascade drives metastatic prostate cancer by coordinately activating Ras and nuclear factor-kappaB. Nat Med. 2010;16:286-94.

14. Liu D, Ren C, Yuan X, Zhang L, Liu J, Liu Q, Yuan J, Yuan D, Jiang XJ. ALDH1 expression is correlated with pathologic grade and poor clinical outcome in patients with astrocytoma. J Clin Neurosci. 2012;19:1700-5.

15. Wang C, Liu Z, Woo CW, Li Z, Wang L, Wei JS, Marquez VE, Bates SE, Jin Q, Khan J, Ge K, Thiele CJ. EZH2 mediates epigenetic silencing of neuroblastoma suppressor genes CASZ1, CLU, RUNX3, and NGFR. Cancer Res. 2012;72:315-24.

16. Li AM, Dunham C, Tabori U, Carret AS, McNeely PD, Johnston D, Lafay-Cousin L, Wilson B, Eisenstat DD, Jabado N, Zelcer S, Silva M,Scheinemann K, Fryer C, Hendson G, Fotovati A, Hawkins C, Yip S, Dunn SE, Hukin J. EZH2 expression is a prognostic factor in childhood intracranial ependymoma: A Canadian Pediatric Brain Tumor Consortium study. Cancer. 2015;121:1499-507.

17. Kee N, Sivalingam S, Boonstra R, Wojtowicz JM. The utility of $\mathrm{Ki}-67$ and BrdU as proliferative markers of adult neurogenesis. J Neurosci Methods. 2002;115:97-105.

18. Yuan Y, Xiang W, Yanhui L, Ruofei L, Shuang L, Yingjun F, Qiao Z, Yanwu Y, Qing M. Ki-67 overexpression in WHO grade II gliomas is associated with poor postoperative seizure control. Seizure. 2013;22:877-81.

19. Wu Z, Wang Q, Wang L, Li G, Liu H, Fan F, Li Z, Li Y, Tu Y. Combined aberrant expression of Bmil and $\mathrm{EZH} 2$ is predictive of poor prognosis in glioma patients. J Neurol Sci. 2013;335:1916.

20. Kogiku M, Ohsawa I, Matsumoto K, Sugisaki Y, Takahashi H, Teramoto A, Ohta S. Prognosis of glioma patients by combined immunostaining for survivin, Ki-67 and epidermal growth factor receptor. J Clin Neurosci. 2008;15:1198-203.

21. Dunbar E, Yachnis AT. Glioma diagnosis: Immunohistochemistry and beyond. Adv Anat Pathol. 2010;17:187-201.

22. Roesler R, Brunetto AT, Abujamra AL, de Farias CB, Brunetto AL, Schwartsmann G. Current and emerging molecular targets in glioma. Expert Rev Anticancer Ther. 2010;10:1735-51.

23. Wang Q, Wang L, Li D, Deng J, Zhao Z, He S, Zhang Y, Tu Y. Kinesin family member 14 is a candidate prognostic marker for outcome of glioma patients. Cancer Epidemiol. 2013;37:79-84.

24. Reya T, Morrison SJ, Clarke MF, Weissman IL. Stem cells, cancer, and cancer stem cells. Nature. 2001;414:105-11.

25. Dean M, Fojo T, Bates S. Tumour stem cells and drug resistance. Nat Rev Cancer. 2005;5:275-84. 
26. Hermann PC, Bhaskar S, Cioffi M, Heeschen C. Cancer stem cells in solid tumors. Semin Cancer Biol. 2010;20:77-84.

27. Rasper M, Schafer A, Piontek G, Teufel J, Brockhoff G, Ringel F, Heindl S, Zimmer C, Schlegel J. Aldehyde dehydrogenase 1 positive glioblastoma cells show brain tumor stem cell capacity. Neuro Oncol. 2010;12:1024-33.

28. Zeng Y, Yang Z, Xu JG, Yang MS, Zeng ZX, You C. Differentially expressed genes from the glioblastoma cell line SHG-44 treated with all-trans retinoic acid in vitro. J Clin Neurosci. 2009;16:28594.

29. Zeppernick F, Ahmadi R, Campos B, Dictus C, Helmke BM, Becker N, Lichter P, Unterberg A, Radlwimmer B, HeroldMende CC. Stem cell marker CD133 affects clinical outcome in glioma patients. Clin Cancer Res. 2008;14:123-9.

30. Kim E, Kim M, Woo DH, Shin Y, Shin J, Chang N, Oh YT, Kim H, Rheey J, Nakano I, Lee C, Joo KM, Rich JN, Nam DH, Lee J. Phosphorylation of EZH2 activates STAT3 signaling via STAT3 methylation and promotes tumorigenicity of glioblastoma stemlike cells. Cancer Cell. 2013;23:839-52.

31. Suvà ML, Riggi N, Janiszewska $M$, Radovanovic I, Provero P, Stehle JC, Baumer K, Le Bitoux MA, Marino D, Cironi L, Marquez VE, Clément V, Stamenkovic I. EZH2 is essential for glioblastoma cancer stem cell maintenance. Cancer Res. 2009;69:9211-8.

32. Qiu S, Huang D, Yin D, Li F, Li X, Kung H, Peng Y. Suppression of tumorigenicity by microRNA-138 through inhibition of EZH2CDK4/6-pRb-E2F1 signal loop in glioblastoma multiforme. Biochim Biophys Acta. 2013;1832:1697-707.
33. Zhang J, Chen L, Han L, Shi Z, Zhang J, Pu P, Kang C. EZH2 is a negative prognostic factor and exhibits pro-oncogenic activity in glioblastoma. Cancer Lett. 2015;356:929-36.

34. Kleer CG, Cao Q, Varambally S, Shen R, Ota I, Tomlins SA, Ghosh D, Sewalt RG, Otte AP, Hayes DF, Sabel MS, Livant D, Weiss SJ,Rubin MA, Chinnaiyan AM. EZH2 is a marker of aggressive breast cancer and promotes neoplastic transformation of breast epithelial cells. Proc Natl Acad Sci USA. 2003;100:11606-11.

35. Bachmann IM, Halvorsen OJ, Collett K, Stefansson IM, Straume O, Haukaas SA, Salvesen HB, Otte AP, Akslen LA. EZH2 expression is associated with high proliferation rate and aggressive tumor subgroups in cutaneous melanoma and cancers of the endometrium, prostate, and breast. J Clin Oncol. 2006;24:268-73.

36. Ueda T, Aozasa K, Tsujimoto M, Ohsawa M, Uchida A, Aoki Y, Ono K, Matsumoto K. Prognostic significance of Ki-67 reactivity in soft tissue sarcomas. Cancer. 1989;63:1607-11.

37. Veronese SM, Gambacorta M, Gottardi O, Scanzi F, Ferrari M, Lampertico P. Proliferation index as a prognostic marker in breast cancer. Cancer. 1993;71:3926-31.

38. Chaloob MK, Ali HH, Qasim BJ, Mohammed AS. Immunohistochemical expression of Ki-67, PCNA and CD34 in astrocytomas: A clinicopathological study. Oman Med J. 2012;27:368-74. 